\title{
Turnaround size of non-spherical structures
}

\author{
Andrea Giusti* and Valerio Faraoni ${ }^{\dagger}$ \\ Department of Physics and Astronomy, Bishop's University \\ 2600 College Street, Sherbrooke Québec, Canada J1M 1Z7
}

16th July 2019

\begin{abstract}
The turnaround radius of a large structure in an accelerating universe has been studied only for spherical structures, while real astronomical systems deviate from spherical symmetry. We show that, for small deviations from spherical symmetry, the gauge-invariant characterization of the turnaround size using the Hawking-Hayward quasi-local mass and spherical symmetry still applies, to first order in the cosmological perturbation potentials and in the deviations from sphericity. This is the first step to include non-spherical systems in the physics of turnaround.
\end{abstract}

\footnotetext{
*E-mail: agiusti@ubishops.ca

${ }^{\dagger}$ E-mail: vfaraoni@ubishops.ca
} 


\section{Introduction}

The observational discovery that the expansion of the universe currently accelerates [1,2] constitutes a major leap forward in our understanding of the large-scale structure of the cosmos. Within the classical picture of General Relativity (GR), this phenomenon is accounted for by postulating the existence of a mysterious and exotic fluid with negative pressure, referred to as dark energy [3. In the last two decades, several alternatives to the ad hoc dark energy have been proposed, ranging from modified theories of gravity [4] 8 ] to the backreaction of inhomogeneities on the large-scale dynamics [9-11].

In recent years, the notion of turnaround radius [12]15] has attracted much attention for its potential as a tool to test the $\Lambda$ Cold Dark Matter $(\Lambda \mathrm{CDM})$ model of the late time universe [16 19. In an accelerating Friedmann-Lemaître-Robertson-Walker (FLRW) universe, the turnaround radius is the scale at which a spherical shell of test particles can no longer collapse because of the accelerated cosmic expansion. In other words, if we look at the problem of structure formation in a universe filled with dark energy, the collapse of local inhomogeneities is opposed by the accelerated expansion of the cosmos, which suggests the existence of a critical scale (the turnaround radius) beyond which an overdensity can no longer collapse since its selfgravity is overcome by the cosmic expansion. With this perspective, we can provide a more rigorous definition of turnaround radius based on the competition between local and cosmological physics [20]. Working, for simplicity, in the framework of GR and limiting ourselves to the first order in the metric perturbations of a spatially flat FLRW universe dominated by a single dark energy fluid (such that $w \equiv p / \rho \approx-1$ ) and restricting to spherically symmetric perturbations, the Hawking-Hayward quasi-local mass (which reduces to the Misner-Sharp-Hernandez mass [21,22 in the spherical case) is computed for a spherical surface $\tilde{S}$ containing all contributions due to the local perturbations. This quasi-local mass can be split into two contributions 23], namely

$$
\tilde{M}_{\mathrm{MSH}} \simeq m a+\frac{H^{2} \tilde{R}^{3}}{2}
$$

where $a$ is the FLRW scale factor, $H \equiv \dot{a} / a$ is the Hubble parameter, $m$ is the Newtonian mass of the perturbation, and $\tilde{R}$ is the areal radius of the surface $\tilde{S}$. One can clearly interpret the first term in the right hand side of Eq. (1) as the contribution due to the energy of the local perturbation, whereas the second term is due to the cosmological expansion. Hence, a natural definition of turnaround radius is obtained by requiring these two terms to be equal 23. The turnaround radius has been discussed also in alternative theories of gravity (see e.g. [24 [31]), but in this work we focus solely on the standard GR picture.

Thus far, the literature on the turnaround radius has studied only spherical structures. The MisnerSharp-Hernandez mass is defined only in the presence of spherical symmetry and is, therefore, inappropriate to describe even small deviations from sphericity. The Hawking-Hayward mass is a natural generalization of the Misner-Sharp-Hernandez one, to which it reduces in spherical symmetry, and it is used in this work.

Preliminary observational evidence seemed to suggest a violation of the maximum turnaround radius predicted by the $\Lambda$ CDM model [32 34], which was interpreted as preliminary evidence against the standard model of cosmology. This statement was later criticized by the same authors following further analysis [35], due to the fact that realistic celestial structures are not perfectly spherical, as assumed instead in theoretical analyses. This abuse of the spherical approximation introduces an error in the comparison of theoretical predictions with real-life astronomical systems (see Refs. 36 38 for early comments). There is little doubt that deviations from spherical symmetry introduce departures from the results predicted for spherical shells of matter and the present work intends to account for the non-spherical features of local overdensities at the theoretical level, in the approximation in which these deviations are small. With inspiration from recent studies on spheroidal deformations of spherical systems [39, 40, we provide the first step in the characterization of the effect of small, non-spherical perturbations on the notion of turnaround size in GR.

We follow the notation of Ref. [41]. The metric signature is $(-+++)$, and we use units in which the speed of light $c$ and Newton's constant $G$ are unity. 


\section{Hawking-Hayward mass in the post-Newtonian regime}

In order to discuss the effects of non-spherical perturbations of a FLRW geometry on the quasi-local energy, we first discuss the analogous situation for post-Newtonian perturbations of a flat background. Then, in Sec. 3. we transpose our results to a perturbed FLRW space, taking advantage of the fact that the FLRW background is conformally flat, and of the known transformation properties of the Hawking-Hayward quasilocal mass.

The post-Newtonian line element in spherical coordinates $(\eta, r, \theta, \varphi)$ reads

$$
\mathrm{d} s^{2}=-\left(1+2 \phi_{\mathrm{N}}\right) \mathrm{d} \eta^{2}+\left(1-2 \phi_{\mathrm{N}}\right)\left(\mathrm{d} r^{2}+r^{2} \mathrm{~d} \Omega_{(2)}^{2}\right)
$$

where the Newtonian potential is small, $\left|\phi_{\mathrm{N}}\right| \ll 1$ and $\mathrm{d} \Omega_{(2)}^{2}=\mathrm{d} \theta^{2}+\sin ^{2} \theta \mathrm{d} \varphi^{2}$ is the line element on the unit 2-sphere.

The quasi-local Hawking-Hayward mass [42 44] is defined as follows. Let $S$ be a spacelike, closed, orientable, 2-surface. Let $\mathcal{R}$ be the induced Ricci scalar on $S$ and consider the outgoing and ingoing null geodesic congruences emanating from $S$, with expansion scalars $\theta_{( \pm)}$and shear tensors $\sigma_{a b}^{( \pm)}$, respectively. Let $\omega^{a}$ be the projection onto $S$ of the commutator of the null normal vectors to $S$ (referred to as "anholonomicity"). Let $\mu$ be the volume 2 -form of $S$ and $A$ its area. The Hawking-Hayward quasi-local mass is

$$
M_{\mathrm{HH}} \equiv \frac{1}{8 \pi} \sqrt{\frac{A}{16 \pi}} \int_{S} \mu\left(\mathcal{R}+\theta_{(+)} \theta_{(-)}-\frac{1}{2} \sigma_{a b}^{(+)} \sigma_{(-)}^{a b}-2 \omega_{a} \omega^{a}\right) .
$$

The quasi-local mass for the post-Newtonian geometry (2]) was computed in Ref. [23], obtaining

$$
M_{\mathrm{HH}}=\frac{1}{8 \pi} \sqrt{\frac{A}{16 \pi}} \int_{S} \mu\left(\frac{4}{r} \frac{\partial \phi_{N}}{\partial r}+\frac{2}{r^{2}} \cot \theta \frac{\partial \phi_{N}}{\partial \theta}+\frac{2}{r^{2}} \frac{\partial^{2} \phi_{N}}{\partial \theta^{2}}+\frac{2}{r^{2} \sin ^{2} \theta} \frac{\partial^{2} \phi_{N}}{\partial \varphi^{2}}\right)
$$

to first order in the metric perturbations. This expression is gauge-invariant to first order [23].

We now want to study small deviations from spherical symmetry, therefore we assume that the Newtonian potential of the mass distribution is

$$
\phi_{\mathrm{N}}(r, \theta, \varphi)=\phi_{0}(r)+\xi f(r, \theta, \varphi)
$$

where the spherical potential $\phi_{0}(r)$ is slightly perturbed, with

$$
\mathcal{O}(f)=\mathcal{O}\left(\phi_{0}\right)=\mathcal{O}\left(\phi_{\mathrm{N}}\right), \quad 0<\xi \ll 1 .
$$

We have, therefore, two expansions: a geometric expansion of spacetime quantities at the post-Newtonian level, and an expansion in small deviations from sphericity at the Newtonian level. We will take the surface $S$ for the calculation of the Hawking-Hayward mass to be an equipotential surface, $\phi_{\mathrm{N}}=$ const., which consists of a slight modification of a spherical surface $S_{0}$ of radius $r_{0}$ (which is an equipotential surface of the spherical potential $\phi_{0}$ ). In order for the perturbed surface $S$ to stay close to $S_{0}$, one must control the perturbation function $f(r, \theta, \varphi)$ so that it does not blow up. If this is not done, the function $f$ could grow very fast and the modified surface $S$ could be far from $S_{0}$ even when the expansion parameter $\xi$ is small. To avoid such situations, we impose that

$$
\frac{\partial f}{\partial r}=\mathcal{O}(\xi)
$$

We can now use the expansion of the Newtonian potential (5) to obtain

$$
\begin{array}{r}
\frac{4}{r} \frac{\partial \phi_{\mathrm{N}}}{\partial r}+\frac{2}{r^{2}} \cot \theta \frac{\partial \phi_{\mathrm{N}}}{\partial \theta}+\frac{2}{r^{2}} \frac{\partial^{2} \phi_{\mathrm{N}}}{\partial \theta^{2}}+\frac{2}{r^{2} \sin ^{2} \theta} \frac{\partial^{2} \phi_{\mathrm{N}}}{\partial \varphi^{2}}= \\
\frac{4}{r} \frac{\mathrm{d} \phi_{0}}{\mathrm{~d} r}+\xi\left(\frac{2}{r^{2}} \cot \theta \frac{\partial f}{\partial \theta}+\frac{4}{r} \frac{\partial f}{\partial r}+\frac{2}{r^{2}} \frac{\partial^{2} f}{\partial \theta^{2}}+\frac{2}{r^{2} \sin ^{2} \theta} \frac{\partial^{2} f}{\partial \varphi^{2}}\right)
\end{array}
$$


to first order in $\xi$ (in the Newtonian expansion) and in $\phi_{\mathrm{N}}$ (in the post-Newtonian expansion).

To proceed, we will now show that the surface of integration $S$ can be replaced by the unperturbed surface $S_{0}$ up to terms of order $\xi^{2}$.

Let us begin with a Newtonian argument to show that $S$ can be replaced by the 2 -sphere $S_{0}$ in the Newtonian expansion without affecting the integral (4) that we want to calculate to first order in the parameter $\xi$ and in the metric perturbations $\phi_{\mathrm{N}}$. By perturbing the sphere $S_{0}$, one adds to the spherical mass density $\rho_{0}(r)$ a density distribution $\delta \rho(r, \theta, \varphi)$, with

$$
\rho(r, \theta, \varphi)=\rho_{0}(r)+\xi \delta \rho(r, \theta, \varphi) .
$$

Correspondingly, the Poisson equation (which can be obtained as the Newtonian approximation of the Einstein equations for the post-Newtonian geometry [23])

$$
\triangle \phi=4 \pi \rho,
$$

with $\triangle$ denoting the Laplace operator, splits into the zero and first order equations in $\xi$

$$
\begin{aligned}
\triangle \phi_{0} & =4 \pi \rho(r), \\
\triangle f & =4 \pi \delta \rho(r, \theta, \varphi) .
\end{aligned}
$$

The total Newtonian mass $M$, which coincides with the Hawking-Hayward mass $M_{\mathrm{HH}}$ to first order, is

$$
\begin{aligned}
M & =\int_{V} \mathrm{~d}^{3} x \rho=\int_{V} \mathrm{~d}^{3} x \rho_{0}+\xi \int_{V} \mathrm{~d}^{3} x \delta \rho \\
& =\frac{1}{4 \pi}\left(\int_{V} \mathrm{~d}^{3} x \triangle \phi_{0}+\xi \int_{V} \mathrm{~d}^{3} x \triangle f\right) \\
& =\frac{1}{4 \pi}\left(\int_{S} \mathrm{~d}^{2} x \boldsymbol{n} \cdot \nabla \phi_{0}+\xi \int_{S} \mathrm{~d}^{2} x \boldsymbol{n} \cdot \nabla f\right),
\end{aligned}
$$

where $V$ is the 3 -volume enclosed by the surface $S$ with unit normal $\boldsymbol{n}=\boldsymbol{\nabla} \phi /|\boldsymbol{\nabla} \phi|$ (and, correspondingly, we denote by $V_{0}$ the 3 -volume enclosed by the 2 -sphere $S_{0}$ with unit normal $\boldsymbol{n}_{0}=\boldsymbol{\nabla} \phi_{0} /\left|\boldsymbol{\nabla} \phi_{0}\right|$ ), and we have used the Gauss theorem in the last line. The second integral in Eq. (13), which is weighted by a factor $\xi$, can be replaced with an integral over the unperturbed surface $S_{0}$ by introducing only an error of order $\mathcal{O}\left(\xi^{2}\right)$. The first integral requires a more detailed discussion.

Let us begin by computing the unit normal $\boldsymbol{n}$ to $S$ to first order:

$$
\begin{aligned}
n & =\frac{\boldsymbol{\nabla} \phi}{|\boldsymbol{\nabla} \phi|}=\frac{\boldsymbol{\nabla} \phi_{0}+\xi \boldsymbol{\nabla} f}{\sqrt{\left(\boldsymbol{\nabla} \phi_{0}+\xi \boldsymbol{\nabla} f\right) \cdot\left(\boldsymbol{\nabla} \phi_{0}+\xi \boldsymbol{\nabla} f\right)}} \\
& \simeq \frac{\boldsymbol{\nabla} \phi_{0}+\xi \boldsymbol{\nabla} f}{\left|\boldsymbol{\nabla} \phi_{0}\right| \sqrt{1+2 \xi \frac{\boldsymbol{\nabla} \phi_{0}}{\mid \boldsymbol{\nabla} \phi_{0}} \cdot \frac{\boldsymbol{\nabla} f}{\boldsymbol{\nabla} \phi_{0} \mid}}} \\
& \simeq \frac{\boldsymbol{\nabla} \phi_{0}+\xi \boldsymbol{\nabla} f}{\left|\boldsymbol{\nabla} \phi_{0}\right|\left(1+\xi \boldsymbol{n}_{0} \cdot \frac{\boldsymbol{\nabla} f}{\left|\boldsymbol{\nabla} \phi_{0}\right|}\right)} \\
& \simeq\left(\frac{\boldsymbol{\nabla} \phi_{0}}{\left|\boldsymbol{\nabla} \phi_{0}\right|}+\xi \frac{\boldsymbol{\nabla} f}{\left|\boldsymbol{\nabla} \phi_{0}\right|}\right)\left(1-\xi \boldsymbol{n}_{0} \cdot \frac{\boldsymbol{\nabla} f}{\left|\boldsymbol{\nabla} \phi_{0}\right|}\right) \\
& \simeq \boldsymbol{n}_{0}\left(1-\xi \boldsymbol{n}_{0} \cdot \frac{\boldsymbol{\nabla} f}{\left|\boldsymbol{\nabla} \phi_{0}\right|}\right)+\xi \frac{\boldsymbol{\nabla} f}{\left|\boldsymbol{\nabla} \phi_{0}\right|} .
\end{aligned}
$$


The integrand in the first integral of Eq. (13) is then

$$
\begin{aligned}
\boldsymbol{n} \cdot \boldsymbol{\nabla} \phi_{0} & \simeq \boldsymbol{n}_{0} \cdot \boldsymbol{\nabla} \phi_{0}+\xi\left[-\left(\boldsymbol{n}_{0} \cdot \boldsymbol{\nabla} f\right)\left(\boldsymbol{n}_{0} \cdot \frac{\boldsymbol{\nabla} \phi_{0}}{\left|\boldsymbol{\nabla} \phi_{0}\right|}\right)+\frac{\boldsymbol{\nabla} f}{\left|\boldsymbol{\nabla} \phi_{0}\right|} \cdot \nabla \phi_{0}\right] \\
& =\boldsymbol{n}_{0} \cdot \boldsymbol{\nabla} \phi_{0}+\xi\left[-\left|\boldsymbol{n}_{0}\right|^{2}\left(\boldsymbol{n}_{0} \cdot \boldsymbol{\nabla} f\right)+\boldsymbol{\nabla} f \cdot \boldsymbol{n}_{0}\right] \\
& =\boldsymbol{n}_{0} \cdot \boldsymbol{\nabla} \phi_{0}+\mathcal{O}\left(\xi^{2}\right) .
\end{aligned}
$$

The equation of the unperturbed surface $S_{0}$ (an equipotential surface of the unperturbed potential) is $\phi_{0}(r)=$ const, or $r=r_{0}$. The surface $S$ is an equipotential surface of the perturbed potential

$$
\phi_{0}(r)+\xi f(r, \theta, \varphi)=\text { const. }
$$

The points of $S$ are located at radius $r=r_{0}+\delta_{1} r$, with $\delta_{1} r / r_{0}=\mathcal{O}(\xi)$. Therefore, the equation (16) describing $S$ can be written as

$$
\phi_{0}\left(r_{0}+\delta_{1} r\right)+\xi f\left(r_{0}+\delta_{1} r, \theta, \varphi\right)=\text { const. }
$$

and expanded to first order in $\xi$ as

$$
\phi_{0}\left(r_{0}\right)+\left.\frac{\mathrm{d} \phi_{0}}{\mathrm{~d} r}\right|_{r_{0}} \delta_{1} r+\ldots+\xi f\left(r_{0}, \theta, \varphi\right)+\left.\xi \frac{\partial f}{\partial r}\right|_{\left(r_{0}, \theta, \varphi\right)} \delta_{1} r+\ldots=\text { const. }
$$

Since $\phi_{0}\left(r_{0}\right)$ and $\mathrm{d} \phi_{0} /\left.\mathrm{d} r\right|_{r_{0}}$ are constants, Eq. (17) yields, to first order,

$$
f\left(r_{0}, \theta, \varphi\right)=\text { const. }
$$

In other words, as for $S_{0}$, the surface $S$ can be described by letting only the polar coordinates $\theta$ and $\varphi$ vary. This means that, to first order in $\xi$, the integrals over $S$ in Eq. (13) can be replaced by integrals over the unperturbed surface $S_{0}$. By combining this result with Eq. (15), Eq. (13) becomes

$$
4 \pi M=\int_{S_{0}} \mathrm{~d}^{2} x \boldsymbol{n}_{0} \cdot \nabla \phi_{0}+\xi \int_{S_{0}} \mathrm{~d}^{2} x \boldsymbol{n}_{0} \cdot \nabla f .
$$

We now use the assumption (77) that $f$ varies gently in the radial direction, whcih implies that the second integral in the right hand side of Eq. (20) is of order $\mathcal{O}\left(\xi^{2}\right)$ and can be dropped. (For special perturbations in which the perturbation potential does not depend on the radial coordinate, $f=f(\theta, \varphi)$, this integral is identically zero since $\boldsymbol{n}_{0} \cdot \boldsymbol{\nabla} f=\mathrm{d} f / \mathrm{d} r=0$.) As a result,

$$
M_{\mathrm{HH}}=\frac{1}{4 \pi} \int_{S_{0}} \mathrm{~d}^{2} x \boldsymbol{n}_{0} \cdot \boldsymbol{\nabla} \phi_{0}+\mathcal{O}\left(\xi^{2}\right) \equiv M_{0}+\mathcal{O}\left(\xi^{2}\right)
$$

where $M_{0}$ is the mass associated with the unperturbed spherical configuration. One can make sense of this result by realizing that the total mass $M=M_{0}+\delta M$ is obtained by adding to the mass $M_{0}$ an additional mass $\delta M$ of order

$$
\delta M \sim \delta V \xi \delta \rho \sim\left(4 \pi r_{0}^{2} \delta_{1} r\right) \xi \delta \rho=\mathcal{O}\left(\xi^{2}\right)
$$

\section{Hawking-Hayward mass in a perturbed FLRW cosmos}

We now turn our attention to the problem of the turnaround radius in cosmology. We assume an accelerated FLRW background universe perturbed by inhomogeneities described by metric perturbations $\phi_{\mathrm{N}}$ of spatial 
extent much smaller than the Hubble radius $H^{-1}$. It is convenient to adopt the conformal Newtonian gauge in which the line element assumes the form

$$
\mathrm{d} \tilde{s}^{2}=a^{2}(\eta)\left[-\left(1+\phi_{\mathrm{N}}\right) \mathrm{d} \eta^{2}+\left(1-\phi_{\mathrm{N}}\right)\left(\mathrm{d} r^{2}+r^{2} \mathrm{~d} \Omega_{(2)}^{2}\right)\right],
$$

where $\phi_{\mathrm{N}}(\boldsymbol{x})$ describes the perturbation and $\eta$ denotes the conformal time of the FLRW background. In this gauge, the line element is explicitly conformal to the post-Newtonian geometry (2). The Hawking-Hayward mass $\widetilde{M}_{H H}$ contained in a topological 2-sphere $S$ in the spacetime (23) was computed in Ref. [23] in two ways. The first method employs the transformation property of the Hawking-Hayward quasi-local mass under the conformal transformation of the spacetime metric $g_{a b} \rightarrow \tilde{g}_{a b}=\Omega^{2} g_{a b}$. The result is [45]

$$
M_{\mathrm{HH}} \rightarrow \widetilde{M}_{\mathrm{HH}}=\sqrt{\frac{\tilde{A}}{A}} M_{\mathrm{HH}}+\frac{1}{4 \pi} \sqrt{\frac{\tilde{A}}{4 \pi}} \int_{S} \mu\left[h^{a b}\left(\frac{2 \nabla_{a} \Omega \nabla_{b} \Omega}{\Omega^{2}}-\frac{\nabla_{a} \nabla_{b} \Omega}{\Omega}\right)-\frac{\nabla^{c} \Omega \nabla_{c} \Omega}{\Omega^{2}}\right],
$$

where $h_{a b}$ is the 2-metric induced on the 2-surface $S$ by the post-Newtonian metric $g_{a b}$ of Eq. (2) and $\nabla_{a}$ is the covariant derivative of $g_{a b}$. The conformal factor $\Omega=a(\eta)$ depends only on the conformal time $\eta$ of the FLRW background. Assuming that the 2-surface $S$ is a small deformation of an unperturbed 2-sphere $S_{0}$ of the Minkowski background of $g_{a b}$ (in which $\phi_{\mathrm{N}}$ is a spherical potential $\phi_{0}(r)$ plus a non-spherical deviation $\xi f(r, \theta, \varphi)$ parametrized by $\xi$ ) with radius $R$, the conformal transformation maps $S_{0}$ into a comoving 2sphere $\widetilde{S}_{0}$ of areal radius $\widetilde{R}=a R$, while $\widetilde{S}$ (i.e., the conformal cousin of $S$ ) is obtained by introducing small non-spherical deformations of $\widetilde{S}_{0}$ parametrized by $\xi$. The Hawking-Hayward mass contained in $\widetilde{S}$ is 23 ,

$$
\widetilde{M}_{\mathrm{HH}}=\Omega M_{\mathrm{HH}}+\frac{R \Omega_{, \eta}}{4 \pi}\left(\int_{\tilde{S}_{0}} \mu \phi_{\mathrm{N}, \eta}-\frac{\Omega_{, \eta}}{\Omega} \int_{\tilde{S}_{0}} \mu \phi_{\mathrm{N}}\right)+\frac{R^{3} \Omega_{, \eta}^{2}}{2 \Omega}
$$

to first order in the perturbations $\phi_{\mathrm{N}}$. Since, to first order in $\phi_{\mathrm{N}}$, the Hawking-Hayward mass is gaugeindependent, this result is manifestly gauge-invariant to this order although it is derived using the conformal Newtonian gauge (23) 11 The mass (25) can also be calculated directly from the post-FLRW metric (23), obtaining the same result $[23]$.

\section{Turnaround size of a large structure}

If the metric perturbation $\phi_{\mathrm{N}}$ is spherically symmetric, $\phi_{\mathrm{N}}=\phi_{0}(r)$, then the Hawking-Hayward mass reduces to 20,23

$$
\widetilde{M}_{\mathrm{HH}}=m a+\frac{H^{2} \widetilde{R}^{3}}{2}\left(1-\phi_{\mathrm{N}}\right) \simeq m a+\frac{H^{2} \widetilde{R}^{3}}{2},
$$

where $m$ is the Newtonian mass computed from the post-Newtonian line element (2) and $\widetilde{R}=a(\eta) R$ is the areal radius of the sphere $\widetilde{S}_{0}$. In the last equality on the right hand side of Eq. (26), the correction $-\phi_{\mathrm{N}} \widetilde{R}(H \tilde{R})^{2}$ is neglected for structures of size $\widetilde{R}$ much smaller than the Hubble radius (this term is of second order in $\left.\widetilde{R} / H^{-1}\right)$.

The current literature on the turnaround radius is restricted to this spherical situation [12, 15, 24 31]. In this context, the turnaround radius is obtained when the "local" and the "cosmological" contributions to the right hand side of Eq. (26) are equal [20], which yields

$$
\widetilde{R}_{c}=\left(\frac{2 m a}{H^{2}}\right)^{1 / 3} .
$$

Other definitions of turnaround radius exist in the literature. For example in Ref. 24, which considers scalar-tensor gravity, the author considers timelike radial geodesics and sets the radial acceleration to zero

\footnotetext{
${ }^{1}$ See also the discussion in Ref. [23].
} 
to define the turnaround radius. In the case of scalar-tensor gravity considered in that reference, there is no accepted definition of Hawking-Hayward mass, which therefore cannot be used. In our article, restricted (at least for the moment) to GR, we prefer to define the turnaround radius using the Hawking-Hayward mass and its splitting in two parts. This definition has the advantage of being covariant and gauge-invariant (the same cannot be said, at least a priori, for the alternative definition of Ref. [24]).

In an accelerated FLRW universe dominated by dark energy with equation of state $P_{\mathrm{DE}}=w \rho_{\mathrm{DE}}$, the (unperturbed) Friedmann equation

$$
H^{2}=\frac{8 \pi}{3} \rho_{\mathrm{DE}}
$$

where $\rho_{\mathrm{DE}}=\rho_{0} a^{-3(1+w)}$ for $w \neq-1$, gives $a(t)=a_{*} t^{\frac{2}{3(1+w)}}$ in terms of the comoving time $t$ of the background (related to $\eta$ by $\mathrm{d} t=a \mathrm{~d} \eta$ ). These relations then yield

$$
\widetilde{R}_{\mathrm{c}}(a)=\left(\frac{3 m}{4 \pi \rho_{0}} a^{3 w+4}\right)^{1 / 3} \equiv \widetilde{R}_{\mathrm{c}}^{(0)} a^{w+4 / 3} .
$$

In terms of the redshift factor $z=\frac{a_{0}}{a}-1$, one has

$$
\widetilde{R}_{\mathrm{c}}(z)=\frac{\widetilde{R}_{\mathrm{c}}^{(0)} a_{0}^{w+4 / 3}}{(1+z)^{w+4 / 3}} .
$$

If the turnaround radius could be determined accurately by astronomical observations, it would be possible to obtain information about the equation of state parameter of dark energy as a function of redshift

$$
w(z)=-\frac{4}{3}+\frac{\ln \left(\widetilde{R}_{\mathrm{c}}^{(0)} a_{0}^{w+4 / 3} / \widetilde{R}_{\mathrm{c}}\right)}{\ln (1+z)} .
$$

The latter could then be used to constrain $w$ if $m a$ and $\widetilde{R}_{\mathrm{c}}$ were known.

Let us consider now more realistic situations in which matter is not distributed spherically and the metric perturbation is described by $\phi_{\mathrm{N}}(\boldsymbol{x})=\phi_{0}(r)+\xi f(r, \theta, \varphi)$ with $\xi \ll 1$ in Eq. (23). Correspondingly, the 2sphere $S_{0}$ is perturbed and its conformal cousin $\widetilde{S}_{0}$ in the perturbed FLRW space (23) is slightly deformed. However, to first order in the parameter $\xi, M_{\mathrm{HH}}$ does not change. In the right hand side of Eq. (25) it is $\phi_{\mathrm{N}, \eta}=0$ and the second term

$$
\frac{R \Omega_{, \eta}^{2}}{4 \pi \Omega} \int_{\tilde{S}_{0}} \mu \phi_{N} \approx \frac{\dot{a}^{2} a R}{4 \pi} 4 \pi R^{2} \phi_{\mathrm{N}} \approx(H \widetilde{R})^{2} \widetilde{R} \phi_{\mathrm{N}}
$$

is of second order in $\widetilde{R} / H^{-1}$ and, therefore, negligible for structures of size $\widetilde{R}$ much smaller than the Hubble radius $H^{-1}$, such as those observed in practice in attempts to determine the critical radius [32 35. As a result, the Hawking mass

$$
\widetilde{M}_{\mathrm{HH}} \simeq m a+\frac{H^{2} \widetilde{R}^{3}}{2}
$$

corresponding to the metric perturbation $\phi_{\mathrm{N}}=\phi_{0}(r)+\xi f(r, \theta, \varphi)$ coincides, to order $\mathcal{O}(\xi)$, with the HawkingHayward mass corresponding to $\phi_{0}(r)$. As a consequence, the definition of turnaround radius obtained by equating the two terms on the right hand side of Eq. (33) is insensitive to the change $\phi_{0}(r) \rightarrow \phi_{0}(r)+$ $\xi f(r, \theta, \varphi)$, to first order in $\xi$. Therefore, when one examines a cosmic structure that deviates from sphericity due to the $\xi$-corrections in the metric perturbation $\phi_{\mathrm{N}}=\phi_{0}(r)+\xi f(r, \theta, \varphi)$, the changes to the definition and the value of the turnaround radius are negligible (to first order in $\xi$ ). This result constitutes a first step toward obtaining cosmological information using astronomical determinations of the turnaround size of cosmic structures which, realistically, depart from perfect sphericity. 


\section{Conclusions}

There is by now little doubt that the use of theoretical predictions for the turnaround size of cosmic structures made under the assumption that the latter are spherical introduces a significant error when comparing theory and observations. All theoretical studies of the turnaround radius thus far have assumed spherical symmetry, while celestial systems are not spherical. Current attempts to pin down the turnaround size with astronomical observations would be helped considerably if the theory took into account deviations from spherical symmetry. As a first step in this direction, we have developed the idea that, given the size of the error that is deemed acceptable from the observational point of view, deviations from spherical symmetry can be neglected in the estimate of the turnaround size only if the post-Friedmannian potential describing the local inhomogeneity in the metric (23) deviates from spherical by less than this error (quantified by our parameter $\xi$ ).

When larger deviations are allowed, second and higher order effects in the expansion in $\xi$ must be taken into account in both the integrand appearing in the integral of Eq. (25) and in the deformation of the equipotential surface $\widetilde{S}$ used to compute the quasi-local mass. This more refined analysis of the HawkingHayward mass and the necessary tools will be developed elsewhere.

\section{Acknowledgments}

This work is supported by Bishop's University and by the Natural Sciences and Engineering Research Council of Canada (Grant No. 2016-03803 to V.F.).

\section{References}

[1] S. Perlmutter et al. [Supernova Cosmology Project Collaboration], "Discovery of a supernova explosion at half the age of the Universe and its cosmological implications," Nature 391, 51 (1998) [astro$\mathrm{ph} / 9712212]$.

[2] S. Perlmutter et al. [Supernova Cosmology Project Collaboration], "Measurements of Omega and Lambda from 42 high redshift supernovae," Astrophys. J. 517, 565 (1999) [astro-ph/9812133].

[3] L. Amendola and S. Tsujikawa, Dark Energy, Theory and Observations (Cambridge University Press, Cambridge, 2010).

[4] T. P. Sotiriou and V. Faraoni, " $f(R)$ Theories Of Gravity," Rev. Mod. Phys. 82, 451 (2010) [arXiv:0805.1726 [gr-qc]].

[5] A. De Felice and S. Tsujikawa, " $f(R)$ theories," Living Rev. Rel. 13, 3 (2010) [arXiv:1002.4928 [gr-qc]].

[6] S. Nojiri and S. D. Odintsov, "Unified cosmic history in modified gravity: from F(R) theory to Lorentz non-invariant models," Phys. Rept. 505, 59 (2011) [arXiv:1011.0544 [gr-qc]].

[7] S. Capozziello and M. De Laurentis, "Extended Theories of Gravity," Phys. Rept. 509, 167 (2011) [arXiv:1108.6266 [gr-qc]].

[8] S. Capozziello, M. De Laurentis and V. Faraoni, "A Bird's eye view of f(R)-gravity," Open Astron. J. 3, 49 (2010) [arXiv:0909.4672 [gr-qc]].

[9] T. Buchert, "On average properties of inhomogeneous fluids in general relativity. 1. Dust cosmologies," Gen. Rel. Grav. 32, 105 (2000) [gr-qc/9906015].

[10] S. Räsänen, "Backreaction: directions of progress," Class. Quant. Grav. 28, 164008 (2011) [arXiv:1102.0408 [astro-ph.CO]]. 
[11] K. Bolejko, M. N. Celerier and A. Krasinski, "Inhomogeneous cosmological models: Exact solutions and their applications," Class. Quant. Grav. 28, 164002 (2011) [arXiv:1102.1449 [astro-ph.CO]].

[12] M. T. Busha, F. C. Adams, R. H. Wechsler and A. E. Evrard, "Future evolution of structure in an accelerating universe," Astrophys. J. 596, 713 (2003) [astro-ph/0305211].

[13] V. Pavlidou and T. N. Tomaras, "Where the world stands still: turnaround as a strong test of $\Lambda$ CDM cosmology," JCAP 1409, 020 (2014) [arXiv:1310.1920 [astro-ph.CO]].

[14] V. Pavlidou, N. Tetradis and T. N. Tomaras, "Constraining Dark Energy through the Stability of Cosmic Structures," JCAP 1405, 017 (2014) [arXiv:1401.3742 [astro-ph.CO]].

[15] M. Lapierre-Léonard, V. Faraoni and F. Hammad, "Cosmological applications of the Brown-York quasilocal mass," Phys. Rev. D 96, no. 8, 083525 (2017) [arXiv:1710.06460 [gr-qc]].

[16] S. Bhattacharya and T. N. Tomaras, "Cosmic structure sizes in generic dark energy models," Eur. Phys. J. C 77, no. 8, 526 (2017) [arXiv:1703.07649 [gr-qc]].

[17] M. Cataneo and D. Rapetti, "Tests of gravity with galaxy clusters," Int. J. Mod. Phys. D 27, no. 15, 1848006 (2018) [arXiv:1902.10124 [astro-ph.CO]].

[18] S. Adhikari, J. Sakstein, B. Jain, N. Dalal and B. Li, "Splashback in galaxy clusters as a probe of cosmic expansion and gravity," JCAP 1811, no. 11, 033 (2018) [arXiv:1806.04302 [astro-ph.CO]].

[19] Z. Roupas, "The Gravothermal Instability at all scales: from Turnaround Radius to Supernovae," Universe 5, no. 1, 12 (2019) [arXiv:1809.07568 [gr-qc]].

[20] V. Faraoni, M. Lapierre-Léonard and A. Prain, "Turnaround radius in an accelerated universe with quasi-local mass," JCAP 1510, no. 10, 013 (2015) [arXiv:1508.01725 [gr-qc]].

[21] C. W. Misner and D. H. Sharp, "Relativistic equations for adiabatic, spherically symmetric gravitational collapse," Phys. Rev. 136, B571 (1964).

[22] W. C. Hernandez and C. W. Misner, "Observer Time as a Coordinate in Relativistic Spherical Hydrodynamics," Astrophys. J. 143, 452 (1966).

[23] V. Faraoni, M. Lapierre-Léonard and A. Prain, "Do Newtonian large-scale structure simulations fail to include relativistic effects?," Phys. Rev. D 92, no. 2, 023511 (2015) [arXiv:1503.02326 [gr-qc]].

[24] V. Faraoni, "Turnaround radius in modified gravity," Phys. Dark Univ. 11, 11 (2016) [arXiv:1508.00475 [gr-qc]].

[25] S. Bhattacharya, K. F. Dialektopoulos and T. N. Tomaras, "Large scale structures and the cubic galileon model," JCAP 1605, no. 05, 036 (2016) [arXiv:1512.08856 [gr-qc]].

[26] S. Bhattacharya, K. F. Dialektopoulos, A. E. Romano, C. Skordis and T. N. Tomaras, "The maximum sizes of large scale structures in alternative theories of gravity," JCAP 1707, no. 07, 018 (2017) [arXiv:1611.05055 [astro-ph.CO]].

[27] S. Nojiri, S. D. Odintsov and V. Faraoni, "Effects of modified gravity on the turnaround radius in cosmology," Phys. Rev. D 98, no. 2, 024005 (2018) [arXiv:1806.01966 [gr-qc]].

[28] S. Capozziello, K. F. Dialektopoulos and O. Luongo, "Maximum turnaround radius in $f(R)$ gravity," Int. J. Mod. Phys. D 28, no. 03, 1950058 (2018) [arXiv:1805.01233 [gr-qc]].

[29] R. C. C. Lopes, R. Voivodic, L. R. Abramo and L. Sodré, "Turnaround radius in $f(R)$ model," JCAP 1809, no. 09, 010 (2018) [arXiv:1805.09918 [astro-ph.CO]]. 
[30] R. C. C. Lopes, R. Voivodic, L. R. Abramo and L. Sodré, "Relation between the Turnaround radius and virial mass in $f(R)$ model," arXiv:1809.10321 [astro-ph.CO].

[31] O. Contigiani, V. Vardanyan and A. Silvestri, "Splashback radius in symmetron gravity," Phys. Rev. D 99, no. 6, 064030 (2019) [arXiv:1812.05568 [astro-ph.CO]].

[32] J. Lee, S. Kim and S. C. Rey, "A Bound Violation on the Galaxy Group Scale: The Turn-Around Radius of NGC 5353/4," Astrophys. J. 815, no. 1, 43 (2015) [arXiv:1511.00056 [astro-ph.CO]].

[33] J. Lee and G. Yepes, "Turning Around along the Cosmic Web," Astrophys. J. 832, no. 2, 185 (2016) [arXiv:1608.01422 [astro-ph.CO]].

[34] J. Lee, "Estimating the Turn-Around Radii of Six Isolated Galaxy Groups in the Local Universe," Astrophys. J. 856, no. 1, 57 (2018) [arXiv:1709.06903 [astro-ph.CO]].

[35] J. Lee, "On the Universality of the Bound-Zone Peculiar Velocity Profile," Astrophys. J. 832, no. 2, 123 (2016) [arXiv:1603.06672 [astro-ph.CO]].

[36] J.D. Barrow and J. Silk, "The Growth of Anisotropic Structures in a Friedman Universe", Astrophys. J., 250, 432 (1981).

[37] J.D. Barrow and P. Saich, "Growth of large-scale structure with a cosmological constant", Mon. Not. Roy. Astron. Soc. 262, 717 (1993).

[38] J.D. Barrow and G. Götz, "Newtonian No-hair Theorems", Class. Quantum Grav. 6, 1253 (1989).

[39] R. Casadio, A. Giusti and R. Rahim, "Horizon Quantum Mechanics for spheroidal sources," EPL 121, no. 6, 60004 (2018) [arXiv:1801.05621 [gr-qc]].

[40] R. Rahim, A. Giusti and R. Casadio, "The marginally trapped surfaces in spheroidal spacetimes," Int. J. Mod. Phys. D 28, no. 01, 1950021 (2018) [arXiv:1804.11218 [gr-qc]].

[41] R. M. Wald, General Relativity (Chicago University Press, Chicago, 1984).

[42] S. Hawking, "Gravitational radiation in an expanding universe," J. Math. Phys. 9, 598 (1968).

[43] S. A. Hayward, "Quasilocal gravitational energy," Phys. Rev. D 49, 831 (1994) [gr-qc/9303030].

[44] S. A. Hayward, "Gravitational energy in spherical symmetry," Phys. Rev. D 53, 1938 (1996) [grqc/9408002].

[45] A. Prain, V. Vitagliano, V. Faraoni and M. Lapierre-Léonard, "Hawking-Hayward quasi-local energy under conformal transformations," Class. Quant. Grav. 33, no. 14, 145008 (2016) [arXiv:1501.02977 [gr-qc]]. 